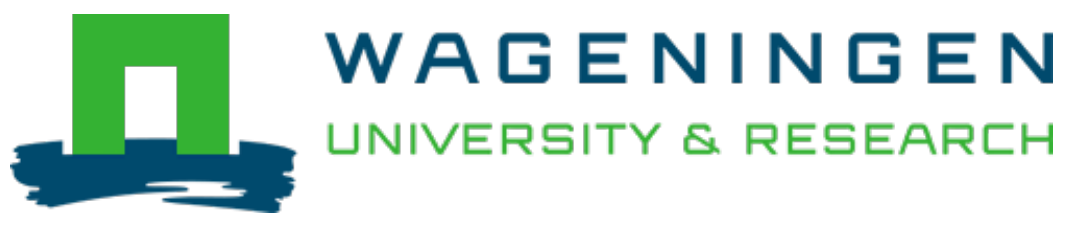

\title{
Automated spider mite damage detection on tomato leaves in greenhouses
}

Acta Horticulturae

Nieuwenhuizen, A.T.; Kool, J.; Suh, H.K.; Hemming, J.

https://doi.org/10.17660/ActaHortic.2020.1268.21

This article is made publicly available in the institutional repository of Wageningen University and Research, under the terms of article $25 \mathrm{fa}$ of the Dutch Copyright Act, also known as the Amendment Taverne. This has been done with explicit consent by the author.

Article $25 \mathrm{fa}$ states that the author of a short scientific work funded either wholly or partially by Dutch public funds is entitled to make that work publicly available for no consideration following a reasonable period of time after the work was first published, provided that clear reference is made to the source of the first publication of the work.

This publication is distributed under The Association of Universities in the Netherlands (VSNU) 'Article $25 \mathrm{fa}$ implementation' project. In this project research outputs of researchers employed by Dutch Universities that comply with the legal requirements of Article $25 \mathrm{fa}$ of the Dutch Copyright Act are distributed online and free of cost or other barriers in institutional repositories. Research outputs are distributed six months after their first online publication in the original published version and with proper attribution to the source of the original publication.

You are permitted to download and use the publication for personal purposes. All rights remain with the author(s) and / or copyright owner(s) of this work. Any use of the publication or parts of it other than authorised under article $25 \mathrm{fa}$ of the Dutch Copyright act is prohibited. Wageningen University \& Research and the author(s) of this publication shall not be held responsible or liable for any damages resulting from your (re)use of this publication.

For questions regarding the public availability of this article please contact openscience.library@,wur.nl 


\title{
Automated spider mite damage detection on tomato leaves in greenhouses
}

\author{
A.T. Nieuwenhuizen ${ }^{a}$, J. Kool, H.K. Suh and J. Hemming \\ Wageningen University and Research, Wageningen, The Netherlands.
}

\begin{abstract}
Spider mites are one of the most challenging pests in the greenhouse, and their management is more and more challenging due to resistance to pesticides. Spider mites prefer young leaves at the outer canopy, stay at the bottom of leaves, and can crawl to nearby plants. Typical symptoms are small yellow and white spots on the upper side of the leaf due to chlorophyll depletion, leading to bronzing of the leaves. The challenge is to detect the spider mite damage in an early stage, as it is hardly possible to detect the actual spider mites underneath the leaves. Based on a structured requirement list, the following camera systems were identified as detection system candidates. Three multispectral cameras and one RGB camera were further investigated in the research. A lab study with hyperspectral imaging was performed that identified discriminating wavebands between healthy and diseased leaves. The wavebands 700,716 and $747 \mathrm{~nm}$ were selected and used in the multispectral systems. Using Fisher LDA, accuracies up to 0.88 could be reached at pixel level with these multispectral cameras. However, none of the setups could meet the spatial resolution to detect spider mite damage at early stage on the leaves. Second step was to perform color image analysis. An identical accuracy of 0.88 with sufficient spatial resolution was reached. Based on these results, a high resolution RGB camera was mounted on a measurement cart. Experiments were performed in a greenhouse compartment with an ongoing spider mite damage infestation in a tomato crop. The results show the automated pipeline for spider mite damage detection in a time series of four recordings over three months, having an accuracy of 0.90 . Heat maps of the infestation rate are presented to the grower and are used for input in models for integrated pest management and to support the decision on the release of beneficial insects.
\end{abstract}

Keywords: greenhouse, spider mite, deep learning, machine vision, pest detection

\section{INTRODUCTION}

Spider mites (Tetranychus urticae) feed on the bottom side of crop leaves. They pierce the chloroplast containing cells and thereby the leaf chlorophyll content is decreased (Sances et al., 1979). Spider mites are one of the challenging pests in greenhouses with tomato crops as their control is increasingly difficult due to resistance to pesticides and difficulties in biological control. The mites prefer young canopy at the outside of the plants for feeding, with canopy damage as a consequence (Herrmann et al., 2012). Typical symptoms are small yellowish white spots on the upper side of the leaf due to chlorophyll depletion, which develop into irregularly shaped white or greyish colored spots, and yellowing and bronzing of leaves may result. Literature on spectral imaging indicates that detection of spider mite itself can be a challenging task due to its small size and crawling on the leaves. However, detection of spider mite damage by means of imaging would be feasible in practice.

Yang et al. (2009) reported that a multispectral radiometer was able to separate between green bug and Russian wheat aphid damage to wheat at the canopy scale. They showed a potential of using multispectral imaging for mite damage detection. Herrmann et al. (2012) indicated that greenhouse pepper and bean leaves were spectrally measured using sub-leaf resolution. They concluded that two-spotted spider mite damage could be spectrally

aE-mail: ard.nieuwenhuizen@wur.nl 
detectable in the visible and near-infrared spectral regions, and five spectral bands are enough to detect damaged leaves. Herrmann et al. (2017) further confirmed that hyperspectral high spatial resolution cameras in a greenhouse or field enable damage detection in an early stage. Several recent studies reported that hyperspectral imaging is useful for the plant disease detection (Mahlein, 2016). A recent study of Fuentes et al. (2017) showed that a deeplearning-based approach is a promising solution to detect diseases and pests in tomato plants, using images taken by a simple camera. They concluded that their proposed system can effectively recognize nine different types of diseases and pests, with the ability to deal with complex scenarios from a plant's surrounding area.

The objectives of this paper are to describe the options of different camera detection techniques to detect spider mite damage on tomato plant leaves, perform lab and greenhouse experiments, analyze results and to draw conclusions including suggestions for improvements.

\section{MATERIALS AND METHODS}

\section{Camera system requirements}

The following is a summary of the camera requirements for spider mite damage detection in a tomato greenhouse crop: 1) the camera system should robustly detect spider mite damage of the leaves. Robust means comparable to human scouting performance; 2) the detection system should handle the highly variable greenhouse environment; 3) the output should be heat maps like produced by human scouting in the greenhouse; 4) the system should be mobile and transportable through the crop and greenhouse compartments. Based on these requirements, state of the art cameras that are commercially available were listed as candidates for the detection system.

\section{Hyperspectral, multispectral and RGB camera}

The first camera considered was the Silios CMS-V multispectral camera (CMS-V, Silios Technologies, Peynier, France) with a range of 550-800 nm with eight custom spectral bands and a resolution of $1280 \times 1024$ pixels. The second camera was the PixelCam multispectral camera (PixelCam, PixelTeq, Largo, USA) with range of 400-1000 nm with eight custom spectral bands and a resolution of $2048 \times 2048$ pixels. The third camera was the SpectroCam multispectral camera (SpectroCam, PixelTeq, Largo, USA) with eight custom spectral bands and a resolution of $1408 \times 1044$ pixels. The fourth camera was a red, green, blue (RGB) color camera (UI-3000SE, IDS, Obersulm, Germany) with a resolution of $4104 \times 3006$ pixels. Hyperspectral cameras were not considered as a viable option in this project, however, the Specim V10E hyperspectral camera (V10E, Specim, Oulu, Finland) with a range of 400-1000 $\mathrm{nm}$ with spectral bands of $2.8 \mathrm{~nm}$ was used to determine discriminative wavebands in a lab experiment for the multispectral cameras.

\section{Lab experiment setup to select discriminating wavebands}

To determine the discriminating wavebands suitable for the multispectral cameras and to determine the performance of detecting the spider mite damage with the RGB camera, spider mite damaged leaves of tomato plants were collected. These leaves were collected in an experimental greenhouse at Proefcentrum Hoogstraten in Belgium (May 10, 2017) and in an experimental greenhouse at Wageningen University and Research in the Netherlands (October 23, 2017). An example image of a damaged leaf is shown in Figure 1. After collecting the leaves they were brought to Wageningen to the lab where the hyperspectral measurements were performed to gather the spectra of the leaves. After gathering the hyperspectral data, analyses were performed with Matlab (Matlab, Mathworks, Natick, USA) and the perClass toolbox (perClass Toolbox, perClass, Delft, The Netherlands). Damage areas were manually labeled and annotated. For the classification Fisher linear discriminant analysis (LDA) was used. Based on the hyperspectral analysis interchangeable stock filters were inserted in the SpectroCam camera to be used for taking images of the leaves. The multispectral images were analyzed with Matlab and perClass toolbox as well. 


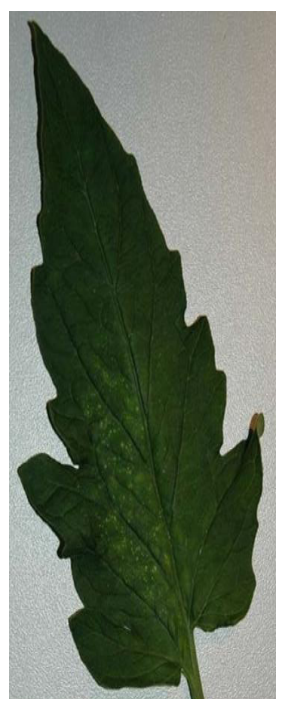

Figure 1. Spider mite damaged leave used for lab experiment.

Using a seven megapixel digital camera RGB images were taken as well of the spider mite damages leaves to determine the detection chances of this technique as well. The RGB images were analyzed with Matlab and perClass toolbox as well.

\section{Greenhouse experiment setup}

To determine the performance of spider mite damage detection under greenhouse growing conditions, a measurement setup was prepared to take images from a standing crop in a greenhouse compartment. The greenhouse compartment was situated at experimental station Hoogstraten in Belgium. Within the 18 row greenhouse compartment a spider mite infestations was initiated such that the damage evolution could be followed during the growth season. During a three month period from May to July 2018 every two weeks manual scoring of spider mite damage as well as camera detection of spider mite damage was performed. Within the greenhouse compartment 10 rows containing 240 numbered plants were monitored and imaged. The number of images was on average 800 per measurement day. The manual scoring of disease level was done at plant level, by counting the number of spider mites on the top leaves of the tomato plants.

The camera setup as used in the greenhouse consisted of several components as shown in Figure 2. A triggering and battery powered supply box was used to enable wheel encoder based image recording every $250 \mathrm{~mm}$ along the crop rows. An IDS UI-3000SE RGB camera with a resolution of $4104 \times 3006$ pixels was used with a lens with a fixed focal length of $25 \mathrm{~mm}$. On both sides of the camera a VIGI-Lux MVS 5002 Machine Vision Strobe flashlight (PerkinElmer Optoelectronics, Salem, USA) was mounted to ensure a constant light level during image acquisition. In combination with a short shutter time of the camera of 50 microseconds this eliminates the effects of changing environmental light conditions and the effects of vibrations of the measurement setup. Images were stored as raw uncompressed 10bit tiff files, to have maximum detail information available.

\section{Spider mite damage detection algorithm}

When all images of the four measurement days were available, analysis on the images was performed. A preliminary investigation showed very high variability in green color brightness off the leaves and crop stand with respect to the camera positioning. Therefore, static methods for image processing did not fit well to detect spots with spider mite damage on the leaves. We therefore chose a deep learning approach to identify spots in images with spider mite damage. 


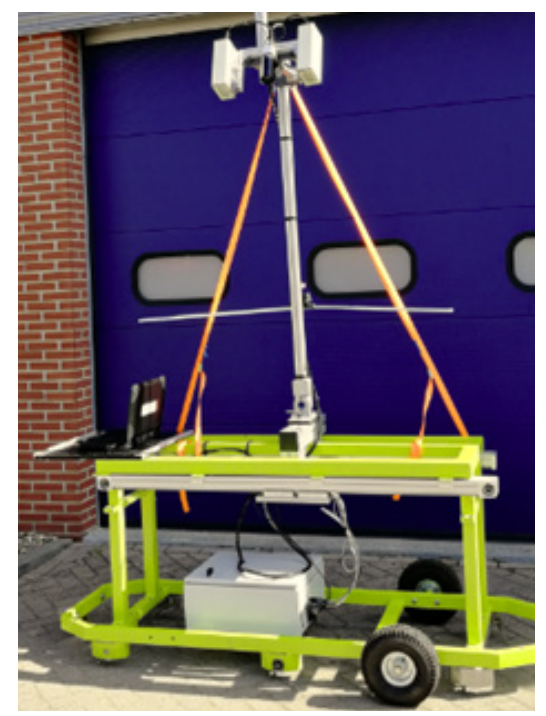

Figure 2. Measurement cart with battery, electronics and camera end flash in the top.

As a result of overlapping images, parts of images were not useful, as there were none, or only very far from the camera, leaves in the image. Therefore, we selected images containing enough green plant material in the following manner. We loaded the images using Python 3.6 and OpenCV 2.0 and did split the image in its three channels R, G and B. A classical excessive green filter was deployed, that is, a pixel was considered excessive green if the value of $2 \mathrm{G}-\mathrm{R}-\mathrm{B}$ was between 80 and 255 . That way a mask was created and after a closing filter we determined the percentage of excessive green pixels in each image. We selected those images that had more than $25 \%$ of the pixels excessive green. The number of images selected varied a lot per day: on the subsequent days there were 402, 320, 101 and 27 files selected. These files were randomly distributed over a training, test and validation set, comprising respectively 60,20 and $20 \%$ of the images. The images of the training and validation set were tiled in a grid with tiles of size $299 \times 299$ pixels. From each of the images of the training and validation sets of the third recording day tiles were selected that were either manually classified as damaged or healthy. The corresponding coordinates and tiles were stored on disk, resulting in 753 training and 358 validation files. A classifier was created using PyTorch (version 0.4) and Torchvision modules, and transfer learning. We retrained the Resnet18 (He et al., 2015; Huang et al., 2016) using transfer learning as explained by Chilamkurthy (2019). On the validation set, the network achieved an accuracy of 0.90 and the confusion matrix is shown in Table 1. Using the trained network the selected images of the 4 days were analyzed. This time each image was tiled with tiles of size $224 \times 224$ pixels. The model classified all the tiles as either healthy or damaged, numerically 0 or 1 respectively. For each tile the percentage of excessive green pixels was recorded, with the same algorithm as above. To analyze the spider mite damage per plant, the images of one row were distributed over 24 groups, as this was the number of plants per row. We considered each group of images as taken from one plant, and defined the infection degree per plant to be:

$$
\frac{1}{\text { number of files }} \sum_{\text {files }} \sum_{\text {tiles }} \text { prediction } * \text { percentage green }
$$

We normalized all infection values by dividing by 56.62, the maximum value determined. 
Table 1. Confusion matrix for spider mite damage detection algorithm.

\begin{tabular}{lcc}
\hline & Predicted healthy & Predicted damaged \\
\hline Healthy & 203 & 14 \\
Damaged & 21 & 120 \\
\hline
\end{tabular}

\section{RESULTS AND DISCUSSION}

\section{Lab experiment}

The camera selection criteria list, together with the literature review resulted in a list of camera systems used in the lab experiment. Within the lab experiment, hyperspectral measurements were performed to identify wavebands that were primarily responsible for the discrimination between healthy and spider mite damaged tomato plant leaves.

The wavebands 700,716 and $747 \mathrm{~nm}$ were selected and used in the multispectral systems. Using Fisher LDA, accuracies up to 0.88 could be reached at sub-leaf area with these multispectral cameras. However none of the setups could meet the spatial resolution to detect spider mite damage at early stage on the leaves. For an application in the greenhouse such cameras, due to the limited spatial resolution, would allow to only acquire images of very small patches of the canopy per image and moreover images will get out of focus very easily for such small patches. In case of the PixelCam camera, that uses a filter wheel to position subsequently the different spectral filters in front of the camera optics, it would require that the measurement system stand completely still during the acquisition of the full image set. Having plants that always move a little by e.g., wind in the greenhouse and a camera that is mounted high up in the crop on a long pole which is sensitive to movements and vibrations this turned out to be not a viable option. As machine vision color cameras with very high resolution are available off the shelf, the second stage in the lab experiment was to perform color image analysis. An identical accuracy of 0.88 with sub-millimeter spatial resolution was reached in discriminating healthy and damaged spots smaller than a square millimeter. Based on these results, a high resolution RGB color camera was preferred to be used on the measurement cart.

\section{Greenhouse experiment}

A measurement setup and cart was successfully realized and used for taking measurements in a greenhouse, shown in Figure 3. An image processing pipeline was developed having an accuracy of 0.90 in detecting spider mite damage on individual crop images as shown in Figure 4. In total 850 images resulted from the selection procedure, shown in Figure 5. Grey locations indicate that there were no images selected. For the images that were automatically analyzed, a visual investigation indicated that the method performed to some extent in providing heat maps in correspondence with human counting.

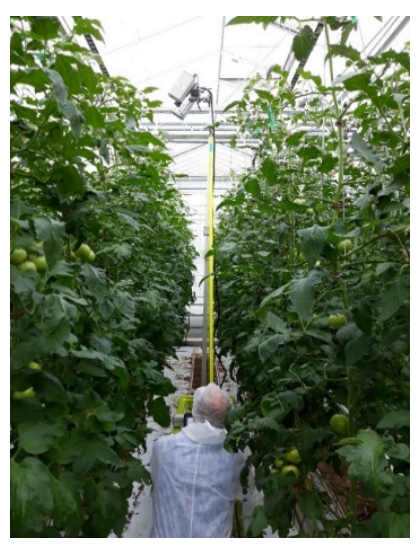

Figure 3. Image recording in greenhouse. 


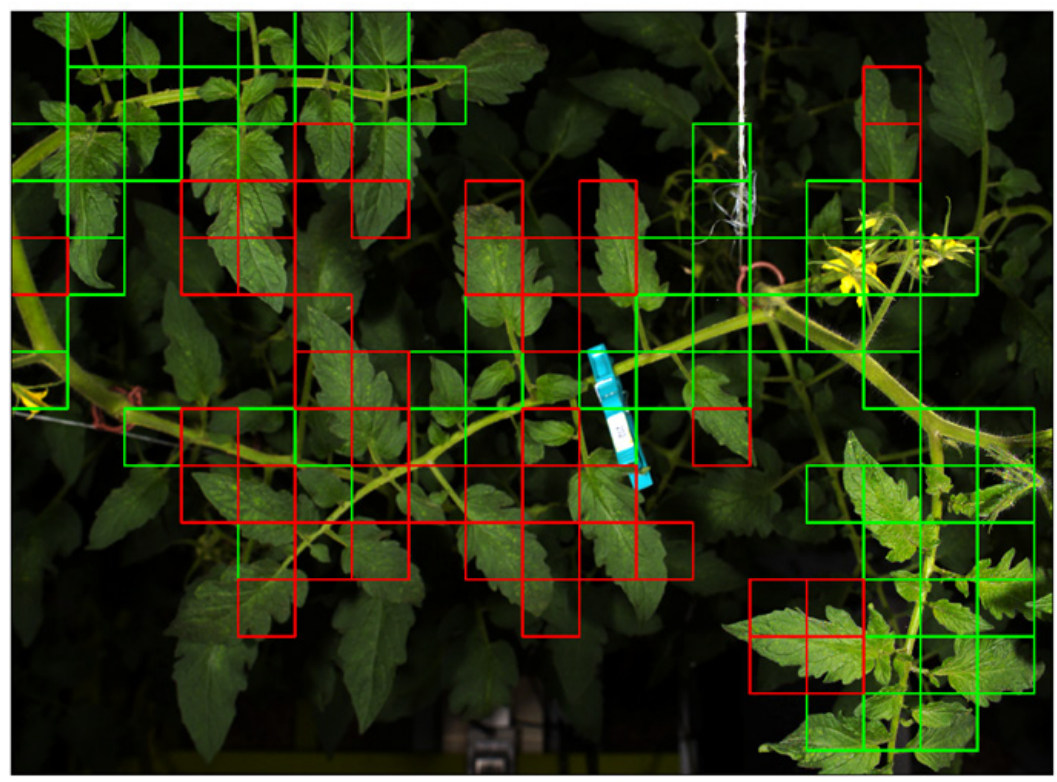

Figure 4. Example original image with the detection results, the represented tiles all contain more than $25 \%$ excessive green pixels. The red tiles are classified as damaged, green tiles as undamaged. Image brightness has been increased by $55 \%$ to enhance reader visibility.

However, the total number of plants with analyzed data was too low to justify a quantitative analysis. The decreasing number of selected images over time was due to the variability in growth among the plants. Also, the tomatoes were grown using a high-wire system, where plants are lowered several times during the season.

\section{CONCLUSIONS}

The lab experiments and camera selection procedure showed that we succeeded in identifying wavebands that can discriminate between healthy and spider mite damaged leaf areas. State of the art multispectral cameras were investigated for suitability. However, these are not suitable for greenhouse application due to limited spatial resolution and standstill requirements. A measurement cart was realized that allows semi-automatic acquisition of high resolution images of the top of tomato plants in the real world greenhouse. It works independent from the environmental light conditions such as night to full-sunlight conditions. Working with images from the greenhouse compared with images in the lab faces more challenges. Locating objects of interest in the images, handling the changing light conditions, handling not standing still plants and vibrating cameras are all additional challenges to reach proof of concept technology readiness. These challenges were faced and overcome in this research. Finally, an automated pipeline to create heat maps to be used by decision support systems for integrated pest management was established.

In future work we expect to investigate the use of 3-dimensional information, for example by stereo imaging, to be able to compensate for the huge variation in image intensity levels caused by the high variability of the camera to plant distance. We foresee to mount the system on a fully autonomous crop-scouting cart to enable better integration with monitoring for integrated pest management. 

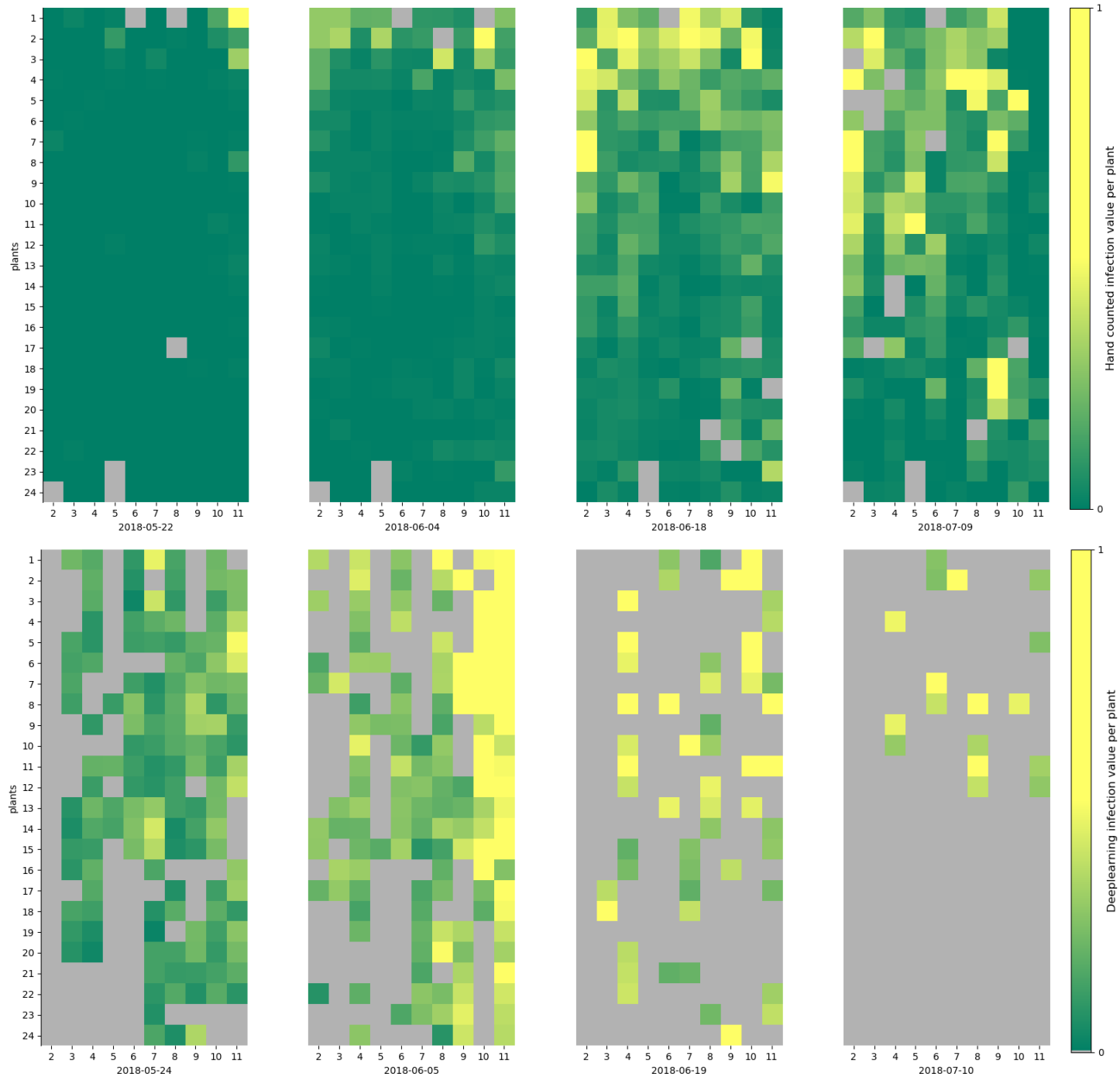

Figure 5. Heat maps produced by normalizing and merging all data. The top four are the hand counted rates. The heat maps are a top view of the glasshouse, in which there where 10 rows each containing 24 plants.

\section{ACKNOWLEDGEMENTS}

We want to thank colleagues from Belgium and Spain within the PeMaTo EuroPep project for providing the plant material, for providing access to the research greenhouse and for the manual pest counting. This work is part of the ERANet research programme C-IPM and the project PeMaTo EuroPep. The work presented here was funded by the Dutch research organization NWO (ALW.FACCE.5) and the Dutch Topsector Tuinbouw en Uitgangsmaterialen (EU2017-06).

\section{Literature cited}

Chilamkurthy, S. (2019). Transfer Learning Tutorial. https://pytorch.org/tutorials/beginner/transfer_ learning_tutorial.html (accessed January 7, 2019).

Fuentes, A., Yoon, S., Kim, S.C., and Park, D.S. (2017). A robust deep-learning-based detector for real-time tomato plant diseases and pests recognition. Sensors (Basel) 17 (9), E2022 https://doi.org/10.3390/s17092022. PubMed He, K., Zhang, X., Ren, S., and Sun, J. (2015) Deep Residual Learning for Image Recognition, arXiv:1512.03385v1.

Herrmann, I., Berenstein, M., Sade, A., Karnieli, A., Bonfil, D.J., and Weintraub, P.G. (2012). Spectral monitoring of two spotted spider mite damage to pepper leaves. Remote Sens. Lett. 3 (4), 277-283 https://doi.org/10.1080/ 
01431161.2011 .576709$.

Herrmann, I., Berenstein, M., Paz-Kagan, T., Sade, A., and Karnieli, A. (2017). Spectral assessment of two-spotted spider mite damage levels in the leaves of greenhouse-grown pepper and bean. Biosyst. Eng. 157, 72-85 https://doi.org/10.1016/j.biosystemseng.2017.02.008.

Huang, G., Liu, Z., Van der Maaten, L., and Weinberger, K.Q. (2016). Densely Connected Convolutional Networks arXiv:1608.06993v5.

Mahlein, A.-K. (2016). Present and future trends in plant disease detection. Plant Dis. 100 (2), 241-251.

Sances, F.V., Wyman, J.A., and Ting, I.P. (1979). Morphological responses of strawberry leaves to infestations of two spotted spider mite. J. Econ. Entomol. 72 (5), 710-713 https://doi.org/10.1093/jee/72.5.710.

Yang, Z., Rao, M.N., Elliott, N.C., Kindler, S.D., and Popham, T.W. (2009). Differentiating stress induced by greenbugs and Russian wheat aphids in wheat using remote sensing. Comput. Electron. Agric. 67 (1-2), 64-70 https://doi.org/10.1016/j.compag.2009.03.003. 